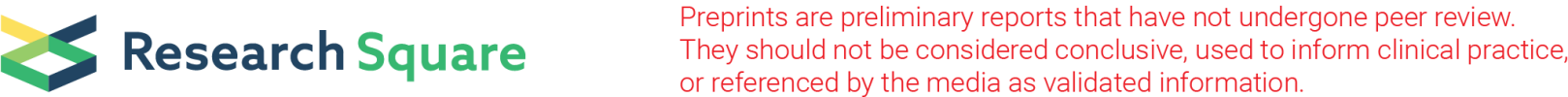

\section{Comparison of Pregnancy Outcomes in Young Patients Following Fresh Versus Frozen Single Blastocyst Transfer: A Retrospective Study}

\section{Yanhong Wu}

the second affiliated hospital of wenzhou medical university

\section{Chang Liu}

the second affiliated hospital of wenzhou medical university

Chaochao Sheng

the second affiliated Hospital of wenzhou medical university

Haitao Xi

the second affiliated hospital of wenzhou medical university

\section{Yanghua Fu}

the second affiliated hospital of wenzhou medical university

Junzhao Zhao ( $\nabla$ z.joyce08@163.com )

the second affiliated hospital of wenzhou medical university

\section{Research article}

Keywords: Single blastocyst transfer, Fresh embryo transfer cycle, Frozen-thawed embryo transfer cycle, Pregnancy outcome, Young patient

Posted Date: August 20th, 2020

DOI: https://doi.org/10.21203/rs.3.rs-48026/v1

License: (c) (1) This work is licensed under a Creative Commons Attribution 4.0 International License. Read Full License 


\section{Abstract}

Background囚Previous studies have shown that in young women, single blastocyst transfer can achieve satisfactory pregnancy results, but there are few studies on the difference between fresh and frozenthawed single blastocyst transfer. In this retrospective analysis, the purpose of this study was to compare the pregnancy outcomes of young patients who received fresh or frozen-thawed single blastocyst transfer, so as to provide further clinical strategies for single blastocyst transfer.

Methods: A retrospective analysis of patients aged $\leq 35$ years old undergoing single blastocyst transfer was performed from January 2018 to December 2018 in the reproductive center of the Second Affiliated Hospital of Wenzhou Medical University. A total of 901 patients were involved and were divided into two groups according to the type of transfer cycle, 693 of which were in the fresh embryo transfer cycle (group A) and 208 of which were in the frozen-thawed embryo transfer cycle (group B). The laboratory and pregnancy outcomes were compared between the groups.

Results囚The number of oocytes retrieved in group B was significantly more than in group $\mathrm{A}(P \otimes 0.05)$. The early miscarriage rate in group $B$ was higher than in group $A$, with significant difference $(P \otimes 0.05)$. There were no significant differences in other basic conditions and obstetric pregnancy outcomes between the two groups.

Conclusions $\mathbb{E}$ Favorable pregnancy outcomes could be obtained in both fresh and frozen-thawed single blastocyst transfer in young patients without increasing the birth defect rate. For patients with risk factors of OHSS such as more oocyte retrieval, ovarian hyper-response, or risk factors of ectopic pregnancy such tubal infertility and the history of ectopic pregnancy, frozen-thawed single blastocyst transfer could be highly recommended in clinical practice. However, since the early miscarriage rate is higher in frozenthawed embryo transfer group, further research is needed to figure out the cause and possible solutions.

\section{Background}

In the past decades, several studies ${ }^{[1,2]}$ have found that human embryos could be cultured to the blastocyst stage in vitro, and successfully obtained clinical pregnancy after blastocyst implantation. With the continuous perfection of cultivation condition in vitro, the blastocyst formation rate has also increased. Compared with embryo transfer in cleavage stage, blastocyst culture is a process of survival of the fittest which shares higher synchronism with endometrial development physiologically, thus increasing embryo implantation rate and clinical pregnancy rate ${ }^{[3]}$. However, with the increasing application of blastocyst transfer in clinical practice, higher clinical pregnancy rate is also accompanied with higher multiple pregnancy rate. How to reduce multiple pregnancy rate while achieving satisfactory pregnancy outcomes has become a research hotspot in recent years. Previous research has uncovered that single blastocyst transfer can achieve similar satisfactory pregnancy outcomes as double cleavagestage embryo transfer ${ }^{[4]}$. Therefore, single blastocyst transfer should be an ideal selection. At present, although quite a lot of researches about the differences of pregnancy outcomes between fresh embryo 
transfer and frozen-thawed embryo transfer have been done, there are few studies aimed at the differences between fresh and frozen-thawed single blastocyst transfer. In view of this, the purpose of this study is to compare the pregnancy outcomes of young patients undergoing fresh or frozen-thawed single blastocyst transfer, thus providing further clinical strategies for single blastocyst transfer.

\section{Methods}

\section{Research objects}

A retrospective analysis of patients undergoing single blastocyst transfer was performed from January 2018 to December 2018 in the reproductive center of the Second Affiliated Hospital of Wenzhou Medical University. The inclusion criteria of this study were: (1)age $\leq 35$ years old; (2) first embryo transfer cycle; (3) normal basal hormone levels. The exclusion criteria include (1) Uterine abnormality under ultrasound such as endometrial polyps, endometrial fibroids, intrauterine adhesion or uterine malformation; (2) endometriosis or adenomyosis; (3) alcoholism or drug addiction. A total of 901 patients were involved and were divided into two groups according to the type of transfer cycle, 693 of which were in the fresh embryo transfer cycle (group A) and 208 of which were in the frozen-thawed embryo transfer cycle (group B).

\section{Fresh Single blastocyst Transfer Cycle}

Long GnRH agonist protocol during early follicular phase or mini-stimulation protocol was adopted as the ovarian stimulation protocol in this study. When 2-3 follicles reached $18 \mathrm{~mm}$ in diameter or one dominant follicle reached $20 \mathrm{~mm}$, intramuscular injection of 4000-10000IU human chorionic gonadotropin (hCG, $10000 \mathrm{IU}$, LIZHU Pharmaceutical Factory, China) at 21:00 to 22:00 that night was executed (trigger). Ultrasound-guided transvaginal oocyte retrieval was performed 36-38 hours after trigger. The retrieved oocytes were subsequently fertilized by IVF/ICSI and incubated at $37^{\circ} \mathrm{C}$ under a humidified gas phase of a mixture of $4 \% 02,6 \% \mathrm{CO} 2$, and $90 \% \mathrm{~N} 2$. Blastocyst scoring was graded according to the Gardner classification system ${ }^{[5]} \llbracket$ Score the expansion stage and incubation status of blastocysts with number 1-6. Score the inner cell mass (ICM) and trophectoderm (TE) with letter A, B or C. A grade of "3BB" or greater was defined as "good quality" and a grade below this was defined as "poor quality". Select a good-quality blastocyst for embryo transfer on the 5th day after oocyte retrieval. If there is no good-quality blastocyst in this cycle, select a blastocyst that is available for embryo transfer. Luteal phase support was implemented with $20 \mathrm{mg}$ dydrogesterone tablets (Duphaston;Solvay Pharmaceuticals B,V. Dose $10 \mathrm{mg} /$ tablet) administered orally twice per day and $200 \mathrm{mg}$ micronized progesterone administered orally or vaginally per day (Utrogestan; Capsugel, Besins Manufacturing Belgium, Bruxelles, Belgium.Dose $0.1 \mathrm{~g} / \mathrm{tablet})$.

\section{Vitrification and Warming Procedures}

Fresh embryo transfer cycle was canceled in patients with ovarian hyperresponse or high risk factors of OHSS. All the embryos were frozen for the next frozen-thawed embryo transfer cycle. The process of 
ovulation trigger, oocyte retrieval and blastocyst grading was consistent with the fresh blastocyst transfer cycle. Blastocysts were vitrified and warmed by the Cryotop method (Kitazato Biopharma) and the operation was carried out in accordance with the kit instructions.

\section{Frozen-thawed Single Blastocyst Transfer Cycle}

Hormone replacement therapy (HRT) or exogenous hormone stimulation with $\mathrm{GnRH}$ agonist ( $\mathrm{GnRH}-\mathrm{a})$ was used for endometrial preparation. HRT patients commenced oral administration of one tablet of estradiol tablet(Femoston;Abbott Biologicals B.V.Dose $2 \mathrm{mg}$ estradiol /tablet)twice a day on the $2^{\text {nd }}-5^{\text {th }}$ day of menstrual cycle. B-ultrasound was performed every 3 to 5 days to measure endometrial thickness and adjust the dosage of estradiol tablets accordingly. When the endometrial thickness $\geq 8 \mathrm{~mm}$ and serum progesterone levels $\varangle 1.5 \mathrm{ng} / \mathrm{ml}$, take $10 \mathrm{mg}$ dydrogesterone tablets (Duphaston;Solvay Pharmaceuticals B,V. Dose $10 \mathrm{mg} /$ tablet) orally twice a day, one tablet of estradiol and dydrogesterone tablet(Femoston;Abbott Biologicals B.V.Dose $2 \mathrm{mg}$ estradiol and $10 \mathrm{mg}$ dydrogesterone/tablet)orally twice a day and $200 \mathrm{mg}$ micronized progesterone administered orally or vaginally twice day(Utrogestan; Capsugel, Besins Manufacturing Belgium, Bruxelles, Belgium.Dose $0.1 \mathrm{~g} /$ tablet).A single i.m. injection of depot GnRH agonist (Decapeptyl ; Ipsen, Milan, Italy.Dose $3.75 \mathrm{mg} / \mathrm{branch}$ ) was administered in the early follicular phase or mid-luteal phase of the cycle. Prepare the endometrium with HRT 4 weeks after GnRHa injection. Select a good-quality blastocyst for embryo transfer on the 5th day after the day of endometrial transfer. If there is no good-quality blastocyst in this cycle, select a blastocyst that is available for embryo transfer. The regimen for luteal phase support was the same as the regimen after endometrial transformation.

\section{Follow-Up}

Serum hCG levels were measured 2 weeks after embryo transfer to determine pregnancy. Another 2 weeks later, clinical pregnancy was identified as the presence of gestational sac and fetal heart beat under transvaginal ultrasound. Besides, Serum $\mathrm{hCG} \geq 15 \mathrm{mlU} / \mathrm{mL}$ without gestational sac 45 days after embryo transfer was defined as biochemical pregnancy. Early miscarriage was defined as the fetal loss before 12 gestational weeks while late miscarriage was defined as the fetal loss after 12 gestational weeks. Premature delivery occurred between 28 to 37 gestational weeks. Neonatal birth weight $<2500 \mathrm{~g}$ was considered as low birth weight infants, while $\geq 4000 \mathrm{~g}$ was fetal macrosomia.

\section{Observation Indicators}

The patients' age, infertility duration, BMI index, cause of infertility, fertilization method, basal hormone levels, the number of oocyte retrieved, proportion of good-quality blastocyst, incidence of moderate to severe OHSS, positive rate of hCG test, clinical pregnancy rate, embryo implantation rate, biochemical pregnancy rate, ectopic pregnancy rate, early miscarriage rate, late miscarriage rate, multiple pregnancy rate, preterm birth rate, live birth rate, neonatal birth weight, fetal sex ratio, birth defect rate and obstetric complications were compared between the groups. 


\section{Statistical methods}

SPSS 22.0 statistical software was used for data analysis. The data were presented as the means \pm SDs, number or percentage. The Student's t-test was applied if the continuous variables were normally distributed. Otherwise, the Dunnetts T3 test was used for the variables of non-normal distribution. The chi-square test and Fisher's exact test were used in data analysis for categorical variables. In this research, $P \otimes 0.05$ was considered statistically significant.

\section{Results}

\section{Comparison of Baseline characteristics and laboratory outcomes}

There were no significant differences in the age, infertility duration, BMI index, cause of infertility, fertilization method, basal hormone levels, proportion of good-quality blastocyst, incidence of moderate to severe OHSS between the two groups $(P>0.05)$. The number of oocytes retrieved in group $B$ was significantly more than in group $\mathrm{A}(P \otimes 0.05)$ (Table 1).

\section{Table 1 Comparison of Baseline characteristics and laboratory outcomes}




\begin{tabular}{|c|c|c|c|}
\hline & Group A $(n=693)$ & Group B $(n=208)$ & $P$ value \\
\hline Age (years) & $29.48 \pm 3.02$ & $29.24 \pm 3.09$ & 0.313 \\
\hline Infertility duration(years) & $3.17 \pm 2.52$ & $3.28 \pm 1.61$ & 0.535 \\
\hline $\mathrm{BMI}(\mathrm{kg} / \mathrm{m} 2)$ & $21.75 \pm 3.09$ & $21.36 \pm 3.38$ & 0.114 \\
\hline Infertility type(\%) & & & 0.976 \\
\hline Primary infertility & $49.64(344 / 693)$ & $49.52(103 / 208)$ & \\
\hline Secondary infertility & $50.36(349 / 288)$ & $50.48(105 / 208)$ & \\
\hline Cause of infertility (\%) & & & 0.480 \\
\hline Tubal factor & $48.20(334 / 693)$ & $42.31(88 / 208)$ & \\
\hline PCOS & 7.36(51/693) & $10.10(21 / 208)$ & \\
\hline Unexplained infertility & $12.55(87 / 693)$ & $11.54(24 / 208)$ & \\
\hline Endometriosis & $3.17(22 / 693)$ & $2.40(5 / 208)$ & \\
\hline Male factor & $16.45(114 / 693)$ & $18.75(39 / 208)$ & \\
\hline \#Multi factors & $12.27(85 / 693)$ & $14.90(31 / 208)$ & \\
\hline Fertilization method (\%) & & & 0.181 \\
\hline IVF & $81.24(563 / 693)$ & $75.96(158 / 208)$ & \\
\hline ICSI & $15.87(110 / 693)$ & $19.23(40 / 208)$ & \\
\hline$|\mathrm{IVF}+| \mathrm{CSI}$ & $2.89(20 / 693)$ & $4.81(10 / 208)$ & \\
\hline \multicolumn{4}{|l|}{ Basal hormone levels } \\
\hline LH(IU/L) & $5.25 \pm 2.47$ & $5.62 \pm 2.71$ & 0.059 \\
\hline FSH(IU/L) & $7.24 \pm 2.04$ & $7.04 \pm 2.15$ & 0.228 \\
\hline $\mathrm{E} 2(\mathrm{pg} / \mathrm{mL})$ & $44.97 \pm 12.31$ & $46.12 \pm 11.65$ & 0.234 \\
\hline$P(n g / m L)$ & $0.57 \pm 0.45$ & $0.58 \pm 0.23$ & 0.698 \\
\hline $\mathrm{PRL}^{\mathrm{a}}(\mathrm{mlu} / \mathrm{L})$ & $10.65(8.71,14.15)$ & $10.58(8.57,15.27)$ & 0.109 \\
\hline No. of oocytes retrieved ${ }^{a}$ & $13.00(10.00,16.00)$ & $17.00(10.00,22.00)$ & $0.001 *$ \\
\hline Proportion of good-quality blastocyst $1 \%$ [ & $88.31(612 / 693)$ & $88.94(185 / 208)$ & 0.803 \\
\hline Incidence of moderate to severe OHSS[\% & $0.43(3 / 693)$ & $0(0 / 208)$ & 0.209 \\
\hline
\end{tabular}

Note: Numbers are mean \pm standard deviation, median(interquartile range) or percentage. 
$\mathrm{BMI}=$ body mass index; $\mathrm{PCOS}=$ polycystic ovarian syndrome; $\mathrm{LH}=$ luteinizing hormone; $\mathrm{FSH}=$ follicle stimulating hormone; $\mathrm{E} 2=$ estradiol; $\mathrm{P}=$ progesterone; $\mathrm{PRL}=$ prolactin; $\mathrm{OHSS}=$ ovarian hyperstimulation syndrome.

\#:Multiple factors defined as more than one reason causing infertility.

a:heterogeneity of variance

*:The No of oocytes retrieved of Group A were significantly difference from those in Group B (P凶0.05).

\section{Coparison of pregnancy outcomes}

The positive rate of hCG test, clinical pregnancy rate, embryo implantation rate, biochemical pregnancy rate, ectopic pregnancy rate, late miscarriage rate, multiple pregnancy rate, preterm birth rate, live birth rate, neonatal birth weight, incidence of macrosomia and low birth weight infants, neonatal death rate, fetal sex ratio, birth defect rate and incidence of obstetric complications were comparable between the two groups ( $\mathrm{P} \otimes 0.05)$. However, the early miscarriage rate in group $B$ was higher than in group $A$, with significant difference( $P \otimes 0.05)$ (Table 2).

\section{Table 2 Coparison of pregnancy outcomes}




\begin{tabular}{|llll|}
\hline & Group A (n=693) & Group B (n=208) & $P$ value \\
\hline Positive rate of hCG test (\%) & $69.26(480 / 693)$ & $70.19(146 / 208)$ & 0.799 \\
\hline Clinical pregnancy rate (\%) & $61.62(427 / 693)$ & $61.54(128 / 208)$ & 0.984 \\
\hline Embryo implantation rate (\%) & $61.62(427 / 693)$ & $61.54(128 / 208)$ & 0.984 \\
\hline Biochemical pregnancy rate(\%) & $7.65(53 / 693)$ & $8.65(18 / 208)$ & 0.637 \\
\hline Early miscarriage rate (\%) & $9.60(41 / 427)$ & $20.31(26 / 128)$ & $0.001 *$ \\
\hline Ectopic pregnancy rate(\%) & $0.70(3 / 427)$ & $0(0 / 128)$ & 1.000 \\
\hline Late miscarriage rate(\%) & $4.22(18 / 427)$ & $3.90(5 / 128)$ & 0.877 \\
\hline Multiple pregnancy rate (\%) & $2.81(12 / 427)$ & $1.56(2 / 128)$ & 0.405 \\
\hline Preterm birth rate (\%) & $5.39(23 / 427)$ & $3.90(5 / 128)$ & 0.502 \\
\hline Live birth rate (\%) & $52.67(365 / 693)$ & $46.63(97 / 208)$ & 0.127 \\
\hline Neonatal birth weight (g) & $3263.92 \pm 571.58$ & $3332.33 \pm 518.38$ & 0.281 \\
\hline Incidence of macrosomia (\%) & $7.75(29 / 374)$ & $7.07(7 / 99)$ & 0.820 \\
\hline Incidence of Low birth weight infants (\%) & $7.49(28 / 374)$ & $5.05(5 / 99)$ & 0.398 \\
\hline Neonatal death rate (\%) & $0.27(1 / 374)$ & $1.01(1 / 99)$ & 0.375 \\
\hline Neonatal sex ratio (\%) & & & 0.455 \\
\hline Male & $51.34(192 / 374)$ & $55.56(55 / 99)$ & \\
\hline Female & $48.66(182 / 374)$ & $44.44(44 / 99)$ & 0.250 \\
\hline Birth defect rate (\%) & $2.14(8 / 374)$ & $2.02(2 / 99)$ & 1.000 \\
\hline Obstetric complications (\%) & & & 3.000 \\
\hline gestational hypertension & $0.23(1 / 427)$ & $0(0 / 128)$ & 0.303 \\
\hline Severe preeclampsia & $6.32(27 / 427)$ & $3.90(5 / 128)$ & \\
\hline Gestational Diabetes & & & \\
\hline
\end{tabular}

*:The Early miscarriage rate of Group A were significantly lower in Group B (P凶0.05).

\section{Discussion}

The application of controlled ovarian hyperstimulation in assisted reproductive medicine makes it possible to obtain sufficient number of oocytes during one menstrual cycle. The number of embryos formed after fertilization often exceeds the number required for one embryo transfer, so the remaining embryos need to be stored frozen, which contributed to the occurrence and development of vitrification 
and warming technology. Frozen embryo transfer cycle enables patients to obtain more opportunities of embryo transfer without repeated oocyte retrieval, thereby avoiding the economic and mental burdens of the patients and maximizing the use of embryos. More importantly, frozen embryos transfer could effectively avoid the occurrence of ovarian hyperstimulation syndrome (OHSS). In a fresh embryo transfer cycle, the process of controlled ovarian hyperstimulation $(\mathrm{COH})$ affects the hormone secretion. High levels of estrogen may lead to early endometrial maturity and affect endometrial receptivity, thus resulting in adverse perinatal and neonatal outcomes ${ }^{[6-7]}$. Although frozen-thawed embryo transfer cycle can avoid hyper stimulation of gonadotropins and provide a better microenvironment for embryo implantation in the uterine cavity ${ }^{[8]}$, the process of embryo freezing may cause blastomere damage, and the impact on maternal and neonatal outcomes still remains controversial.

Osamu Ishihara et al. conducted a retrospective study of 3047 fresh single blastocyst transfer cycles and 11329 frozen-thawed single blastocyst transfer cycles, and found that compared with fresh single blastocyst transfer, frozen-thawed single blastocyst transfer could result in similar pregnancy outcomes without increasing the miscarriage rate ${ }^{[9]}$. However, Daimin Wei et al. have found that the embryo implantation rate, biochemical pregnancy rate and live birth rate of frozen-thawed single blastocyst transfer were significantly higher $(P \otimes 0.05)$ than that of fresh single blastocyst transfer through a large sample, multi-center, randomized controlled clinical study ${ }^{[10]}$. In this study, the enrolled patients were all no more than 35 years old and treated with IVF for the first time, which could effectively avoid the disadvantages of the advanced age, decreased ovarian reserve and repeated implantation failure. The results showed that both fresh single blastocyst transfer and frozen-thawed single blastocyst transfer could achieve satisfactory pregnancy outcomes. Also, there were no significant differences in adverse pregnancy outcomes such as biochemical pregnancy and late miscarriage rate between the two groups. However, it should be noted that the early miscarriage rate of frozen-thawed single blastocyst transfer was significantly higher than that of fresh single blastocyst transfer $(P<0.05)$. This conclusion conflicts with the researches mentioned above, the reason may be that early miscarriage is generally related to the quality of the embryo. Vitrification and warming procedures of the embryo may affect the embryonic activity to varying degrees. Studies have found that the incidence of chromosome abnormalities in frozen-thawed embryos is significantly higher than that in the fresh embryos ${ }^{[11]}$. Although embryo frozenthawed procedures are developing towards higher efficiency and safety, the potential risks are still not negligible.

The incidence of ectopic pregnancy in IVF patients has increased nearly three times compared with normally conceived females, which ranges between $2.1 \% \sim 8.6 \%{ }^{[12-13]}$. The main reason is that tubal infertility accounts for a large part of the infertility. Also, studies have shown that the incidence of ectopic pregnancy in women with tubal infertility resorting to IVF-ET could be up to $11 \%^{[14]}$. Moreover, the number of embryo transferred in the early days is often $\geq 2$. While improving the pregnancy outcomes, it also increases the incidence of heterotopic pregnancy. Compared with embryos in cleavage stage, blastocysts stay in the uterine cavity for a shorter period of time, thereby reducing the probability of migration to the fallopian tube and the occurrence of ectopic pregnancy. Some researchers have concluded that single 
blastocyst transfer in the frozen-thawed cycle could significantly reduce the risk of ectopic pregnancy ${ }^{[9]}$, which indicated that the frozen-thawed cycle might be closer to a natural state ${ }^{[15-16]}$. The increased ectopic pregnancy rate in fresh embryo transfer cycle might be due to the hyperphysiological hormonal environment caused by ovarian stimulation ${ }^{[17]}$. In this study, three cases of ectopic pregnancy occurred in the fresh single blastocyst transfer group, but no case occurred in the frozen-thawed group. The incidence of ectopic pregnancy in both groups was lower than that of natural pregnancy, with no statistical difference ( $P \otimes 0.05$ ), which suggested that single blastocyst transfer could reduce the incidence of ectopic pregnancy. For people with high risk factors such as tubal infertility and previous history of ectopic pregnancy, single blastocyst transfer is recommended, among which the frozen-thawed cycle is more preferred.

OHSS is an iatrogenic complication caused by ovarian hyperstimulation with exogenous gonadotropins, which can be life-threatening in severe cases. The incidence in IVF-ET is about $1-10 \%{ }^{[18]}$. In order to prevent OHSS, some patients choose the freeze-all approach and wait for the next frozen-thawed cycle to reduce the production of endogenous hCG, thereby reducing the generation of vasoactive substances.. In this study, the median number of oocyte obtained in the fresh single blastocyst transfer group was 13 and there were 3 cases of moderate to severe OHSS, while the median number of oocytes obtained in the frozen-thawed group was 17 and no case of moderate to severe OHSS occurred. The number of oocyte retrieved in the two groups was statistically different, which further reminds us that for patients with ovarian hyperresponse or with high risk factors of OHSS, frozen-thawed single blastocyst transfer may be a good choice as it can not only avoid the occurrence of OHSS but also obtain the same satisfactory clinical outcome as in the fresh cycle.

It has been reported that the birth defect rate is higher in the frozen-thawed cycle ${ }^{[19]}$, but some scholars ${ }^{[20]}$ found that the frozen-thawed cycle actually did not significantly increase the birth defect rate in singletons. In this study, the birth defects in the fresh single blastocyst transfer group included cardiac malformations ( 2 cases), hand malformation ( 1 case), pulmonary sequestration ( 1 case), ear malformation ( 1 case), biliary atresia (1 case) and favism (1 case), while in the frozen-thawed group, it was consisted of hypospadias ( 1 case) and congenital torticollis (1 case). There was one case of newborn death in both groups. No statistical differences were found between the two groups in terms of birth defect rate and neonatal death rate $(P>0.05)$, but still lacks of large sample randomized controlled studies to confirm.

With regard to the obstetric complications, the incidence of gestational hypertension in frozen-thawed embryo transfer cycle was significantly increased compared with fresh embryo transfer cycle according to a meta-analysis ${ }^{[21]}$. This study has not yet found that frozen-thawed single blastocyst transfer would increase the risk of obstetric complications. Since whether frozen-thawed cycle would increase the risk of obstetric complications is still controversial, it could not be concluded at present. The meta-analysis also indicated that the incidence of macrosomia and large for gestational age in frozen-thawed embryo transfer cycle was higher than that of fresh embryo transfer cycle, while the incidence of premature, low 
birth weight infants, and small for gestational age were lower in the frozen-thawed cycle. There was a difference in neonatal birth weight between the two groups. Some researchers ${ }^{[22]}$ believed that this might be related to the epigenetic changes during frozen-thawed embryo transfer and maternal hyperestrogenemia during fresh embryo transfer. However, in this study, the neonatal birth weight, neonatal sex ratio, incidence of macrosomia, small for gestational age and preterm birth were comparable between the two groups (Pख0.05). The reason might be that in the process of ovarian stimulation, for patients with ovarian hyperresponse or high risk factors of OHSS, it was usually recommended that patients give up fresh embryo transfer and consider freeze-all approach to reduce the adverse pregnancy outcomes caused by maternal hyperestrogenemia. In addition, single blastocyst transfer also greatly reduce the incidence of multiple pregnancy, thereby further reducing the occurrence of adverse pregnancy outcomes such as preterm birth and small for gestational age. These results provide new ideas for explaining the differences between the frozen-thawed and fresh single blastocyst transfer, which still need further research.

\section{Conclusions}

In 2018, the Chinese Expert Consensus on the number of embryo transferred has proposed the practice of selective single embryo transfer. Given continuous improvement of blastocyst culture, the time for China to implement single blastocyst transfer is mature. Favorable pregnancy outcomes could be obtained in both fresh and frozen-thawed single blastocyst transfer in young patients without increasing the birth defect rate. For patients with risk factors of OHSS such as more oocyte retrieval, ovarian hyper-response, or risk factors of ectopic pregnancy such tubal infertility and the history of ectopic pregnancy, frozenthawed single blastocyst transfer could be highly recommended in clinical practice. However, since the early miscarriage rate is higher in frozen-thawed embryo transfer group, further research is needed to figure out the cause and possible solutions.

\section{Abbreviations}

Ovarian hyperstimulation syndrome OHSS

Follicle-stimulating hormone FSH

Luteinizing hormone LH

Estrogen-2 E2

Progesterone $\mathrm{P}$

Prolactin PRL

Human chorionic gonadotropin hCG

Inner cell mass ICM 
Trophectoderm TE

Body mass index BMI

Hormone replacement therapy HRT

GnRH agonist GnRH-a

Controlled ovarian hyperstimulation $\mathrm{COH}$

In vitro fertilization and embryo transfer IVF-ET

Intra-cytoplasmic sperm injection ICSI

\section{Declarations}

\section{Acknowledgments}

The authors thank everyone participating in the clinical and laboratory work.

\section{Author's contribution}

YHW and CL designed and performed the study, analyzed the data, and wrote and edited the manuscript. JZZ and HTX conceived and participated in the study design, evaluated the results and edited the manuscript. CCS and YHF contributed to data collection and statistical analysis. All authors have read and approved the final manuscript.

\section{Funding}

This study was funded by Wenzhou City Key Innovation Team of Reproductive Genetics Grant, Zhejiang, China (No. C20170007). The Funder had no role in the design, conduct or interpretation of the study. The open access publication fee is paid by the funder.

\section{Availability of data and materials}

The transcripts from which this manuscript was developed are available on request from the corresponding author.

\section{Ethics approval and consent to participate}

This study was approved by the Ethics Committee (Institutional Review Board) of the Second Affiliated Hospital and Yuying Children's Hospital of Wenzhou Medical University and informed written consent was obtained from all participants.

\section{Competing interests}


We declare that we do not have any commercial or associative interest that represents a conflict of interest in connection with the work submitted.

\section{Contributor Information}

Yanhong Wu,the first author,Doctor,Email:330592479@qq.com

Chang Liu, BS, the co-first author, Emial:632027539@qq.com

Chaochao Sheng,Emial:670203310@qq.com

Haitao Xi, MD,Email: xihaitao2001@163.com

Yanghua Fu, Email:ffyyhh20101006@163.com

Junzhao Zhao,the Corresponding author,Email:z.joyce08@163.com

\section{References}

1. Gardner DK, Vella P, Lane M,et al.Culture and transfer of human blastocystes implantation rates and reduces the need for multiple embryo transfer[J].Fertil Steril,1998,69(1):84-88.

2. Jones GM, Trounson AO, Gardner DK, et al. Evolution of a culture protocol for successful blastocyst development and pregnancy[J]. Hum Reprod. 1998;13(1):169-77.

3. Glujovsky D, Farquhar C, Quinteiro Retamar AM, et al. Cleavage stage versus blastocyst stage embryo transfer in assisted reproductive technology[J]. Cochrane Database Syst Rev. 2016;30(6):CD002118.

4. Prados N. Quiroga R,Caligara C,et al.Elective single versus double embryo tranfer:live birth outcome and patient acceptance in a prospective randomised trial[J]. Reprod Fertil Dev. 2015;27:794-800.

5. Gardner DK. Lane M, Stevens J,et al.Blastocyst score affects implantation and pregnancy outcome:towards a single blastocyst transfer[J].Fertil Steril,2000. 73:1155-1158.

6. Kansal Kalra S, Ratcliffe SJ, Milman J,et al. Perinatal morbidity after in vitro fertilization is lower with frozen embryo transfer[J]. Fertil Steril. 2011;95(2):548-53.

7. Pereira N, Elias RT, Christos PJ,et al.Supraphysiologic estradiol is an independent predictor of low birth weight in full-term singletons born after fresh embryo transfer[J].Human Reproduction,2017. 32(7):1410-1417.

8. Barnhart KT.Introduction:are we ready to eliminate the transfer of fresh embryos in in vitro fertilization?[J].Fertil Steril,2014. 102(1):1-2.

9. Ishihara O, Kuwahara A, Saitoh H. Frozen-thawed blastocyst transfer reduces ectopic pregnancy risk: an analysis of single embryo transfer cycles in Japan[J]. Fertil Steril,2011,95(6):1966-1969.

10. Wei D, Liu J-Y, Yun, Sun,et al. Frozen versus fresh single blastocyst transfer in ovulatory women: a multicentre, randomised controlled trial[J]. The Lancet. 2019;393:1310-8. 
11. Chatzimeletiou K, Morison EE,Panagiotidis Y,et al. Cytoskeletal analysis of human blastocysts by confocal laser scanning microscopy folowing vitrification[J]. Hum Reprod. 2012;27:106-13.

12. Talbot K,Simpson R,Price N,et al.Heterotopic pregnancy[J].J Obstet Gynaecol,2011(1):07-12.

13. Kirk E, Papageorghiou AT, Condous G,et al.The diagnostic effectiveness of an initial transvaginal scan in detecting ectopic pregnancy[J].Hum Reprod,2007. 22(11):2824-2828.

14. Refaat B. Dalton E,Ledger WL,et al.Ectopic pregnancy secondary to in virto fertilization-embryo tranfer:pathogenic mechanisms and management strategies[J]. Reprod Biol Endocrinol. 2015;13:300 .

15. Londra L Moreau, Strobino C. D,et al.Ectopic pregnancy after in virto fertilization:differences between fresh and frozen-thawed cycles[J].Fertil Steril,2015. 104(1):110-118.

16. Muller V,Makhmadalieva M,kogan I,et al.[J].Ectopic pregnancy following in virto fertilization:metaanalysis and single-center experience during 6 years[J].Gynecol Endocrinol,2016. 32(sup2):69-74.

17. Acharya KS, Acharya CR, Provost MP,et al.Ectopic pregnancy rate increases with the number of retrieved oocytes in autologous in virto fertilization with non-tubal infertility but not donor/recipient cycles:an analysis of 109140 clinical pregnancies from the Society for Assisted Reprodutive Technology registry[J].Fertil Steril,2015. 104(4):873-878.

18. MacDougall MJ,Tan SL,Jacobs HS.In vitro fertilization and the ovarian hyperstimulation syndrome[J].Human Reproduction,19927(5): 579-600.

19. Belva F, Henriet $S$, Van den Abbeel E,et al.Neonatal outcome of 937 children born after tranfer of cryopreserved embryos obtained by ICSI and IVF and comparision with outcome data of fresh ICSI and IVF[J].Human Reprod,2008. 23(10):2227-2238.

20. Shi W, Xue X, Zhang S,et al.Perinatal and neonatal outcomes of 494 babies delivered from 972 vitrified embryo transfers[J].Fertil Steril,201297(6): 1338-1342.

21. Mageshwari A, Raja EA,Bhattacharya S. Obstetric and perinatal outcomes after either fresh or thawed frozen embryo tranfer: an analysis of 112432 singleton pregnancies recorded in the Human Fertilisation and Embryology Authority anonymized dataset[J]. Fertil Steril. 2016;106:1703-8.

22. Hu XL, Feng $C$, Lin $X H$,et al. High maternal serum estradiol environment in the first trimester is associated with the increased risk of small-for-gestational-age birth[J]. J Clin Endocrinol Metab. 2014;99:2217-24. 